\title{
EL SENTIDO DEL TRABAJO EN MUJERES PRIVADAS DE LIBERTAD EN CHILE ${ }^{1}$
}

\author{
Ana Cárdenas Tomažič \\ ISF München - Alemania \\ ana.cardenas@isf-muenchen.de
}

\author{
Rosario Undurraga R. \\ Universidad Finis Terrae - Chile \\ rosarioundurraga@yahoo.com
}

Recibido: 28-02-2014

Aceptado: 00-00-2014

\section{Resumen}

Este artículo vincula género, trabajo y cárcel para problematizar variadas formas de integración a la sociedad y al mercado laboral. Se analiza el caso de mujeres recluidas por micro-tráfico en el sistema penitenciario chileno. El estudio se basa en 30 entrevistas semi-estructuradas realizadas a reclusas del CPF Santiago durante el año 2013, explorando la trayectoria laboral y la definición de trabajo de mujeres privadas de libertad. Esta investigación visibiliza la situación de mujeres marginadas en múltiples dimensiones y muestra cómo las actividades ilegales y la posición social de estas mujeres cuestionan y reproducen el orden social. Se ilustra así la estandarización del trabajo moderno y modos atípicos de participación laboral, problematizando formas emergentes de integración en el capitalismo flexible.

Palabras claves: cárcel, género, orden social, reclusas, trabajo.

\begin{abstract}
This paper explores the relation between gender, work and incarceration by problematizing the various ways in which women seek to integrate into the labour market. We analyse the case of women prisoners in Chile who have been convicted of micro-trafficking. The methodology is based on 30 semi-structured interviews with women prisoners in Santiago in 2013. We explore their work trajectories and their definitions of work. The study visualizes the situation of marginalized women in multiple dimensions, showing how their illegal activities and their social position (re)produce and contest the social order. The study illustrates the standardization of modern work by highlighting atypical forms of labour participation, and problematizes ways in which women try to integrate themselves under flexible capitalism.
\end{abstract}

Keywords: prison, gender, social order, female inmates, work.

\footnotetext{
${ }^{1}$ Agradecemos la contribución de Magdalena Muñoz Lalanne en el estudio de campo y a la Corporación Abriendo Puertas por facilitarnos el acceso al Centro Penitenciario Femenino (CPF) de Santiago de Chile. Adicionalmente, Ana Cárdenas agradece a la Fundación Alexander von Humboldt por su apoyo en el estudio comparado del empleo penitenciario femenino a nivel internacional.
} 


\section{Introducción}

La población penitenciaria femenina ha aumentado exponencialmente durante las últimas décadas en Chile, superando el crecimiento experimentado por los hombres en el mismo período (Gendarmería de Chile, 2008a, 2008b, 2008c, 2009, 2011). El aumento de la tasa de encarcelamiento en Chile se sitúa por sobre el promedio mundial y latinoamericano (Ungar, 2003; International Centre for Prison Studies, 2012). Según datos de Gendarmería de Chile, las mujeres representan hoy alrededor del $8.6 \%$ de la población condenada a penas privativas de libertad y casi un $13 \%$ del total de imputados que esperan su condena en prisión (Valenzuela et al., 2012). Si se hace una distinción según género y delito, se observa que la población penitenciaria masculina tiende a estar recluida principalmente por robo con intimidación (16\%), mientras que las mujeres por tráfico ilícito de drogas (15.1\%) (Gendarmería de Chile, 2008a). Estas cifras nos llevan a reflexionar sobre la relación entre género, trabajo y cárcel, así como los diversos modos en que el trabajo está siendo entendido en las sociedades contemporáneas.

Este artículo sugiere que las mujeres involucradas en actividades de micro-tráfico estarían cuestionando el orden social, particularmente, el orden de género y la noción de trabajo. Al respecto, proponemos lo siguiente:

Primero, las mujeres reclusas quebrarían el orden de género al no cumplir con las expectativas sociales de género respecto a sus roles de cuidado. En el marco del patriarcado y sus resultantes asimetrías de poder entre hombres y mujeres, la mujer tradicionalmente ha cumplido roles de servicio y ha asumido la responsabilidad del cuidado de otros/as (Arriagada, 2013; Cockburn, 1983; Hughes, 2002; Walby, 1985, 1990). Esta función primordial de cuidado sería propio de una "buena madre". Sin embargo, la mujer privada de libertad atentaría con lo esperado a su género, pues se encuentra imposibilitada de desempeñar sus roles de cuidado a diario, lo que finalmente tendría "efectos colaterales" en su hijos/as (Comfort, 2003).

Segundo, el orden de género se estaría reproduciendo en el mercado laboral ilegal. La segregación laboral sitúa a las mujeres en roles de subordinación y menor reconocimiento en el mercado laboral (Anker, 1997) y con ello reproduce el orden patriarcal de género. De modo similar, en la actividad ilegal de tráfico de estupefacientes las mujeres suelen ocupar las 
posiciones inferiores dentro de la jerarquía de poder en dicha actividad económica. Así, su labor es peligrosa, poco reconocida y les otorga menos recompensas que aquellas labores llevadas a cabo en los puestos más altos de la jerarquía del "negocio" de la droga (Torres Angarita, 2008).

Tercero, las reclusas quebrarían el orden social al cometer un delito. Según la ley 20.000 del Código Penal chileno, el micro-tráfico es considerado un delito. Por tanto, la participación de mujeres en esta actividad implica cometer un acto desviado de la norma social vigente.

Cuarto, las mujeres más pobres y de minorías étnicas son quienes típicamente conforman la población penitenciaria femenina en América Latina (Antony, 2003; Cárdenas, 2011, 2013; DPLF et al., 2003); esto implica que este grupo social vulnerable estaría sobrerrepresentado en la población penitenciaria. Las mujeres privadas de libertad estarían cuestionando el orden social, pues mediante los mayores ingresos que perciben en relación a los trabajos remunerados a los que usualmente pueden acceder modifican la distribución de poder económico en esta sociedad. Al mismo tiempo, las reclusas por micro-tráfico estarían cuestionando el concepto tradicional de trabajo.

En este artículo se problematiza la posición de las mujeres en el mercado laboral en general y en el tráfico de drogas en particular desde una perspectiva de género, es decir, considerando las estructuras y dinámicas de conformación y distribución del poder entre hombres y mujeres (Antony, 2007; Kreisky, 2000; Cárdenas, 2010, 2011, 2013; Torres Angarita, 2008; Undurraga, 2011, 2013). Al respecto, se pone énfasis en la noción de trabajo como manifestación de un cuestionamiento al orden social desde las vivencias y discursos de las reclusas en relación al trabajo. Específicamente, se discute el significado del trabajo para mujeres privadas de libertad, cuestionando el concepto de trabajo en el "capitalismo flexible" (Sennett, 1998, 2005).

La metodología de esta investigación es cualitativa. Se basa en 30 entrevistas semiestructuradas realizadas a mujeres privadas de libertad del Centro Penitenciario Femenino (CPF) de Santiago de Chile durante el año 2013. En las entrevistas se explora la trayectoria laboral y la definición de trabajo de las reclusas. La edad de las mujeres fluctúa entre 19 y 65 años, con un promedio de 39 años. Todas las entrevistas fueron grabadas y transcritas. Las 
participantes son asociadas a un número de identificación (ID) para resguardar su anonimato y confidencialidad.

Los resultados de este estudio muestran las experiencias de mujeres privadas de libertad en torno al trabajo. La contribución de mujeres reclusas es relevante dada su marginalidad múltiple. La interrelación entre género, clase social, etnia, nivel educacional y privación de libertad, entre otras categorías sociales, las confina en un lugar marginal de la sociedad en diversas dimensiones. Su voz ha sido silenciada y su existencia confinada. Esta es una oportunidad para visibilizar las experiencias de personas ampliamente excluidas en la sociedad actual.

\section{2. ¿Qué es trabajo? Las tenues fronteras entre trabajo formal, informal y criminal}

El trabajo ha sido definido en términos de relaciones de mercado, reforzando así su aspecto económico. Más aún, se ha homologado trabajo con empleo. La Organización Internacional del Trabajo (OIT) recomienda una definición internacional y estándar de empleo que lo relaciona con remuneración (LABORSTA, 2014). Las estadísticas nacionales en Chile adscriben los postulados de OIT en su definición de empleo. Por ejemplo, se considera "ocupado" a toda persona de 15 años o más que durante la semana anterior a la toma de la encuesta (semana de referencia) trabajó una hora o más por remuneración o ganancia, trabajó una hora o más por cuenta propia independientes, trabajó 15 horas o más como familiar no remunerado, o no trabajó por encontrarse temporalmente ausente (INE, 2014). Estas definiciones son una base para evaluar las tasas de empleo y desempleo, entre otros indicadores de productividad de un país. Por el contrario, el trabajo no remunerado no es contabilizado como insumo productivo por la UNSNA (United Nations System of National Accounts) y, por lo general, no es medido (Hoskyns y Rai, 2007; Waring, 2008). Entonces, el trabajo que no es medido no es considerado como una contribución oficial a la economía. Esto es, aquellas actividades económicas informales, voluntarias o ilegales no son consideradas como parte de las actividades económicas que está generando ingresos al país. Al respecto, el feminismo ha 
cuestionado la escisión entre trabajo remunerado y no remunerado, y la sociología ha problematizado las fronteras entre el trabajo formal, informal y criminal.

El feminismo cuestiona el significado del trabajo que prima en torno al trabajo asalariado; postula que el trabajo es relacional y que tanto el trabajo productivo como reproductivo son necesarios para que una sociedad pueda marchar. Esto significa que tanto el trabajo realizado en la esfera pública, que es usualmente remunerado, así como el realizado en la esfera doméstica, que en general no es remunerado, son indispensables para que una sociedad exista y se reproduzca. Así, el feminismo se opone a la definición de trabajo restringida a trabajo remunerado únicamente, postulando que el trabajo involucrado en la reproducción social debe ser contabilizado como trabajo y como parte de la economía (Hoskyns y Rai, 2007). Esto es, la reproducción de la vida social depende tanto de lo producido en la esfera pública y economía formal, como del trabajo doméstico y lo no remunerado (Glucksmann, 1990). Por ello, las investigaciones feministas han intentado valorizar el trabajo no remunerado, el trabajo doméstico, el trabajo de cuidado, el trabajo emocional, el trabajo voluntario y el trabajo comunitario.

En la sociología, Portes, Castells y Benton (1989) han hecho un aporte significativo para diferenciar tres tipos de actividades económicas: formal, informal, criminal. Los autores reconocen estos tres tipos de actividades como acciones económicas, diferenciándolas en su modo de producción, distribución y tipo de producto final. Es así como las actividades económicas de tipo criminal se distinguen de los restantes tipos de actividades por su producto final, puesto que éste vulnera las normas sociales escritas. Sin embargo, coincide con el resto de las actividades económicas en su proceso de producción y distribución, es decir, en el grado de legalidad o ilegalidad de las acciones humanas involucradas. En consecuencia, esta tipología de las actividades económicas permite abordar las actividades económicas y su trabajo subyacente desde un concepto de trabajo que supera las fronteras entre el trabajo asalariado y todas aquellas actividades económicas ilícitas, evitando así establecer una jerarquía de base normativa entre dichas acciones (ver Tabla 1). 
Tabla 1: Tipos de actividad económica

\begin{tabular}{|c|c|c|}
\hline $\begin{array}{c}\text { Proceso de producción } \\
\text { y distribución }\end{array}$ & Producto final & Tipo económico \\
\hline Lícito & Lícito & Formal \\
\hline Ilícito & Lícito & Informal \\
\hline Lícito o ilícito & Ilícito & Criminal \\
\hline
\end{tabular}

(Fuente: Portes, 2000)

$\mathrm{Al}$ respecto, la integración de las mujeres al mercado laboral y las condiciones precarias en que aquella ocurre actualmente (OIT, 1998; Piras, 2006; Sassen, 2007a, 2007b; PNUD, 2010) parecieran favorecer la generación de modos de integración alternativos al trabajo formal. Una forma alternativa de lograr el objetivo económico para integrarse a las sociedades latinoamericanas sería a través de actividades ilegales (Arriagada y Hopenhayn, 2000; Portes, Castells y Benton, 1989; Portes, 2000). De hecho, en la sociedad chilena contemporánea el motivo por el cual la mayoría de las mujeres están recluidas en el sistema penitenciario es el tráfico de estupefacientes, es decir, una fuente de ingreso que no proviene de un trabajo formal (Cárdenas, 2010, 2011, 2013). En ocasiones, el micro-tráfico puede constituirse en una estrategia de integración a la sociedad de libre mercado, ya que en ésta el consumo de bienes y servicios se torna un eje central de reconocimiento, independientemente del modo en que se han generado u obtenido los recursos para dicho consumo. El trabajo ilegal/criminal se transforma así en un medio más para alcanzar la integración de las personas y sus familias en dicho contexto (Portes y Hoffman, 2007).

En este artículo nos interesa discutir y visibilizar lo que significa el trabajo para mujeres privadas de libertad. Nos focalizamos en este grupo por su marginalidad múltiple y por la posibilidad de lograr a través de éste una mayor comprensión respecto al sentido del trabajo en las sociedades modernas de "capitalismo flexible". Consideramos que las experiencias de estas reclusas desafían la concepción tradicional que ha primado en la sociedad moderna en torno al trabajo, ofreciendo información empírica de gran relevancia para dicha reflexión.

A continuación desarrollaremos los ejes centrales de lo que significa trabajo para las reclusas. Aquí queremos relevar que existe una diversidad de prácticas sociales y conceptualizaciones de lo que se entiende como trabajo, lo que finalmente incide en las prácticas subjetivas. Por ello, más que abordar la diversidad considerando las características humanas que hacen a las personas diferentes unas de otras y sus variables sociales como son etnia/raza, orientación sexual, edad, clase social, género, etc., la diversidad se manifiesta aquí 
en la pluralidad de formas de concebir lo que es trabajo y construir variadas estrategias de integración en las sociedades contemporáneas.

\section{Te ganarás el pan con el sudor de tu frente: entre subordinación y autonomía}

El proceso de asalarización del trabajo moderno supuso subordinación y la aceptación de determinados deberes laborales con respecto a un empleador, a cambio de un salario estable y el progresivo reconocimiento de determinados derechos sociales (Castel, 1995). Dicho proceso de asalarización se construyó sobre la base de un proceso de socialización que buscó estandarizar los tiempos de las personas, específicamente de los hombres a los tiempos de las empresas y sus procesos de racionalización (Aulenbacher, 2010). Al contrario, las mujeres tendieron a ser socializadas en el marco de los tiempos variables que supone la vida cotidiana (Jurczyk, 2001).

Pese a que el proceso de modernización en América Latina supuso también un proceso creciente de racionalización de la vida económica y con ello, de las diversas esferas de la vida, el trabajo informal que prevaleció en gran parte del continente supuso una lógica de trabajo totalmente contraria, específicamente, la autodeterminación de los objetivos, los tiempos y espacios de trabajo (Carpio, Klein y Novakovsky, 2000; Bayón, Roberts y Saraví, 1998; Portes, 2000; Portes, Castells y Benton, 1989). Dicha tendencia se manifiesta, por ejemplo, en el recuento de la trayectoria laboral de una reclusa de 65 años (ID24). Ella destaca el trabajo informal e independiente como una alternativa viable para compatibilizar su rol de madre y de trabajadora:

"Lo que he hecho en mi vida como trabajo, comerciante ambulante, lo que más he trabajado, y lo demás, porque uno trabaja independiente, trabaja para el que quiere y ahí va viendo. Yo al menos que fui madre, tenía dos hijos y estaba sola con mis hijos. Entonces yo tenía el tiempo de trabajar y de ver a mis hijos y eso era lo más importante. Entonces no tenía patrón, no tenía nada, ni horarios que cumplir. Entonces eso me daba... si yo de tonta no más cuando mis hijos crecieron me fui al norte. Después ya de vieja vine a meterme en esas cosas que no debería de meterme". 
En el caso de mujeres privadas de libertad, muchas de ellas definen lo que es trabajo por las condiciones de trabajo, como por ejemplo, levantarse temprano, esforzarse, cumplir horarios, tener jefatura, responsabilidades, subordinación y acatar órdenes. Estos aspectos parecieran estar vinculados a una moral religiosa cristiana, la que supone que el trabajo implica esfuerzo y sacrificio, como lo indica la cita bíblica: "Te ganarás el pan con el sudor de tu frente" (Génesis 3:19). Aquí, el trabajo es una suerte de castigo para Adán y Eva por desobedecer a Dios y comer del fruto prohibido, siendo expulsados del paraíso. Así, el trabajo se instruye como el medio para poder sobrevivir, el que supone esfuerzo y sacrificio. Al respecto, las participantes atribuyen una connotación negativa a estas características, definiendo incluso al trabajo como "esclavizante". Sin embargo, consideran que éste les entrega cierta estabilidad y un posible acceso a beneficios. Una reclusa de 28 años (ID1) nos dice:

"Trabajar es esforzarte, levantarse temprano, tener una pega por ser buena, que tení un sueldo fijo (...) Lo principal es el esfuerzo y hay que tener las ganas de trabajar, levantarte temprano, ser responsable con tu trabajo. Salir de acá de la sección para llegar a tu trabajo, trabajar las horas que te piden, eso es trabajo".

Subordinación y autodeterminación constituyen entonces dos características intrínsecas al trabajo desde el punto de vista de estas mujeres. Al respecto, si bien las reclusas tienen una variedad de significaciones en torno al trabajo, la mayoría considera que lo que realizaba "en la calle" era su trabajo, lo que cuestiona el concepto tradicional de trabajo. El sentido del trabajo para mujeres recluidas por efecto del micro-tráfico se sustenta entonces como una forma alternativa de integración económica que se distancia de las normas habituales de trabajo. Es decir, sus actividades "en la calle" son una forma alternativa de integración social. Más aún, su discurso se basa en las mismas motivaciones de aquellas personas que trabajan en actividades legales y formales: llevar el sustento al hogar.

En los relatos de las reclusas se manifiesta entonces la tensión entre trabajar en la cárcel y en la calle: el orden externo versus la autorregulación. Desempeñarse laboralmente en la cárcel implica someterse a un orden de tiempos, espacios y a una jerarquía, mientras que el trabajo en la calle destaca por las diversas posibilidades de autorregulación. Así, para estas reclusas el trabajo formal supone subordinación explícita, mientras que en la calle el trabajo lo definiría y organizaría uno mismo, pese a las jerarquías existentes en el negocio del micro- 
tráfico (Torres Angarita, 2008). De esta manera lo expresa una reclusa de 25 años (ID29) al comparar su labor actual en la cárcel con lo que realizaba antes en la calle:

\begin{abstract}
"No va a ser lo mismo. Yo encuentro que aquí es más paqueao que en la calle, porque aquí tení que levantarte temprano, tení que salir cuando limpian, que después te retan, porque andai con pijama. En la calle no, en la calle tú mandai; aquí te mandan, aquí recibí órdenes, aquí recibí lo que gendarmería dice y en la calle no, tú poní tus condiciones, tú poní tus reglas, si lo tomas, si lo dejas, es tu responsabilidad, si quieres salir adelante es tu responsabilidad, si quieres cambiar tú tomas tu decisión".
\end{abstract}

Trabajar suele implicar someterse a las órdenes de un otro y enmarcar las propias acciones cotidianas y biográficas dentro de aquéllas. Tanto en el contexto de la sociedad moderna como en el contexto del capitalismo flexible, trabajar implica un proceso de socialización que supone un cierto grado de estandarización de las acciones de las personas; esto es, trabajar presume asumir un rol, relacionarse con otros, realizar una tarea en un espacio y tiempo determinado, entre otros aspectos. Para muchas reclusas, este tipo de experiencia es relativamente nueva, la que implica un proceso de internalización de normas de comportamiento, como el someterse a la regulación de tiempos, órdenes y actividades. Así lo relata esta reclusa de 30 años (ID8):

“A mí me ha costado mucho trabajar, sí, porque no me gusta que me manden, me gusta mandarme sola; me gusta hacer lo que yo quiera y aquí hay que acatar órdenes. Entonces me ha costado, me ha costado, pero igual llevo como 3 años trabajando. Ya ahora estoy acostumbrada, pero al principio me costaba mucho levantarme temprano, que me dijeran lo que tenía que hacer, que controlaran mis horarios".

En el marco del proceso de precarización laboral, trabajar suele implicar someterse a condiciones de trabajo inestables y que no aseguran un bienestar material básico. Al respecto, un número importante de reclusas asocia el trabajo legal/formal a condiciones laborales caracterizadas por bajos salarios, relaciones de subordinación, largas jornadas, en definitiva, mucho esfuerzo para poca recompensa. Específicamente, perciben al trabajo legal/formal como el camino largo y difícil para obtener dinero, mientras que delinquir y traficar se muestran como la vía fácil y rápida para conseguir lo que materialmente necesitan - a pesar de las 
potenciales consecuencias como estar en la cárcel. Frente a dicho escenario, las reclusas perciben el trabajo ilegal/criminal como una alternativa que implica menor esfuerzo y tiempo: el dinero llega rápido, pero se gasta rápido; se gana fácil y se tiene una vida fácil. Es la inmediatez de las necesidades y su satisfacción mediante el dinero rápido (ilegal) lo que ha solido orientar las acciones de estas mujeres hacia el micro-tráfico. Este es el caso de una mujer privada de libertad de 32 años (ID2):

"He trabajado, pero después a uno le empieza a picar el bichito poh, de ganar plata más fácil, más rápido y más encima yo tengo a mis 3 hijos. Entonces ha salido difícil. Por eso opté como por lo más fácil, pero igual aqui están las consecuencias".

Frente a un mercado laboral que ofrece un escaso bienestar material a estas mujeres y pocas posibilidades de conciliación trabajo productivo-trabajo reproductivo, el tráfico de estupefacientes se vuelve una alternativa de sustento para estas mujeres y sus familias. Por ejemplo, una mujer de 64 años (ID23) se refiere al trabajo y los motivos por los cuales llegó al tráfico de drogas:

"Bueno siempre fue el motivo para poder sobrevivir, porque yo me casé a los 15 años, tuve mis hijas y a los 21 me separé, porque mi marido me pegaba a mí y a mis hijas. Así que me separé y de ahí tuve que empezar a trabajar para poder sobrevivir. Trabajé varios años vendiendo, pero siempre sobreviviendo a duras penas, que todos los meses o me cortaban el agua o me cortaban la luz o no me alcanzaba el dinero para pagar el almacén. Por eso es que me metí en la cuestión de la droga, porque vi plata, no tan fácil, pero vi dinero como nunca había podido tener. Eso fue".

El cuestionamiento al concepto de trabajo asalariado y el desarrollo de actividades de micro-tráfico parecieran estar relacionados con concepciones particulares acerca del consumo, el cual supondría una inmediatez temporal. En cambio, la experiencia de trabajar remuneradamente en la cárcel las ha vinculado de un modo distinto respecto al tiempo, el dinero y el consumo, puesto que primero deben realizar el sacrificio de trabajar antes de poder consumir (antes, cuando micro-traficaban, dicho esfuerzo se traduce a actividades generalmente acotadas en el tiempo y el consumo ocurre entonces de manera inmediata). Así lo expresa una reclusa de 44 años (ID9): 
"Ahora que estoy en este lugar le he tomado como más importancia al trabajo, que ya no es tan fácil como cuando uno sale a robar o a traficar, que la plata es más fácil. No poh, aquí aprendí a cuidar, a tratar de tener por etapas la plata, supongamos pa'l trabajo, pa' la casa. Entonces igual valorai más lo que haci”.

De manera similar, la narración de una mujer privada de libertad de 50 años (ID10) destaca la relevancia del sacrificio, es decir, la postergación del beneficio económico para la valoración del ingreso ganado. Cuando dicho sacrificio no constituye la base moral para el trabajo remunerado, trabajo remunerado y consumo constituirían acciones estructuradas secuencialmente en el presente:

"Una cosa muy importante que ahora aprendi aqui, en este lugar lo aprendi, porque te poni las pilas trabajando y tus monedas las cuidai. No como la de la calle, que moneda fácil la gastai, la gastai, la gastai...acá no poh”.

En conclusión, el sentido del trabajo para las reclusas previo a su período penitenciario suele cuestionar el sentido del trabajo formal o asalariado. Esto, no solo por la subordinación que éste supone, sino por condiciones de trabajo que no asegurarían un bienestar material mínimo para estas mujeres y sus familias. Al respecto, el micro-tráfico constituye un modo de integración crecientemente "legitimado" en el marco del capitalismo flexible, en especial en las sociedades neoliberales latinoamericanas (véase Portes y Hoffman, 2007), en la medida en que sería un camino que permitiría, por lo menos momentáneamente, la integración de las personas al consumo.

En consecuencia, el trabajo asalariado no se constituiría hoy en un referente normativo ampliamente legitimado por parte de la población y con ello, orientador de sus acciones. Más aún, en el caso de mujeres madres que carecen de recursos para delegar, aunque sea parcialmente, sus tareas de cuidado y de proveedoras, el trabajo asalariado y sus condiciones de trabajo crecientemente precarias parecieran ser un horizonte de acción que cada vez más carece de sentido.

\section{El micro-tráfico como oficio}


La construcción de relaciones de mercado para la oferta y demanda de fuerza de trabajo se ha estructurado en las sociedades modernas progresivamente a partir de los oficios y las profesiones. Éstas son formas sociales de capacidades y destrezas entrelazadas entre sí, así como tareas especializadas, tipificadas y delimitadas institucionalmente (Demszky von der Hagen y Voß, 2010; Voß, 1997), las que preestablecen tanto lo que las personas saben y pueden hacer así como lo que no saben o no están autorizadas a hacer (ibid.). Específicamente, los oficios y las profesiones son "formas estandarizadas" de venta de la fuerza de trabajo, es decir, "certificados de capacidades" (Bolte, Brater y Beck, 1988) de las personas para la oferta de sus cualificaciones en el mercado del trabajo (Voß, 1997, 2007). Aquellas tienen como objetivo el logro de las funciones sociales y son asumidas por las personas para percibir ingresos monetarios (ibid.).

A su vez, son "mecanismos decisivos de integración social y posicionamiento de los seres humanos" (Demszky von der Hagen y Voß, 2010: 751), donde la distribución social de las profesiones constituye "una forma básica de orden social y desigualdad establecida sobre la base de una creciente diferenciación de las funciones sociales" que dan origen a diversos privilegios (ibid.). Por último, los oficios y las profesiones tienen un fuerte arraigo nacional y tienen una diversidad de manifestaciones históricas y significados culturales (ibid.) que varían de acuerdo a la división del trabajo resultante de los procesos de transformación económica y técnica así como a las dinámicas de poder (por ejemplo, entre diferentes profesiones u oficios) en un determinado momento (Demszky von der Hagen y Voß, 2010; Voß, 1997). Dentro de los privilegios asociados a las profesiones se encuentran el status, el prestigio, la autonomía y los ingresos (Demszky von der Hagen y Voß, 2010), los que finalmente sitúan a las personas en posiciones diferenciadas de poder dentro de un determinado orden social.

$\mathrm{Al}$ respecto, cabe tener presente que las profesiones y los oficios constituyen un saber y poder específico que han sido diferenciados, especializados y determinados en términos de sus contenidos (Brater, 2010: 805). Más aún, las profesiones y los oficios "no se encuentran simplemente a disposición (de las personas)" (ibid.), sino que deben ser transmitidos y adquiridos por la persona que desea desempeñarse en el marco de éstos a través de un proceso de formación, es decir, "deben ser desarrolladas por medio del aprendizaje” (ibid.). Esto significa, que "el proceso de producción y reproducción de la fuerza de trabajo" debe ser organizado y asegurado socialmente para que el sistema de trabajo y los objetivos económicos 
puedan ser alcanzados (ibid.). En este contexto, el sistema educacional adquiere un rol central en dicho proceso, pues constituye el espacio socialmente construido para cumplir la función de formar a la fuerza de trabajo para asegurar su adecuada participación en el mercado del trabajo. Esto implica no solo transmitir un conjunto de saberes, sino que también disciplinar a los individuos para hacerlos funcionales a los requerimientos del aparato productivo. Dicho objetivo se ha vuelto cada más relevante en la nueva fase del capitalismo "flexible" (Sennett, 1998, 2005), donde la fuerza de trabajo femenina es incorporada crecientemente a los mercados laborales nacionales e internacionales como "una fuerza de trabajo barata y dócil" (Öztimur, 2007: 117) y con ello, adaptable a los requerimientos de las fluctuaciones económicas (véase al respecto Sassen, 2007a; Aulenbacher, 2010).

Aprender un oficio o profesión conllevaría la promesa de poder acceder al mercado laboral y así la ansiada movilidad social. Por el contrario, la falta de estudios puede constituirse en una desventaja central para integrarse al mercado del trabajo de manera satisfactoria. En efecto, las reclusas suelen identificar la falta de educación formal como una de sus principales carencias para participar en el mercado laboral. Por ejemplo, así lo expresa esta mujer privada de libertad de 32 años (ID2):

"Trabajar es sacarse la ñoña por poquita plata, porque de repente cuando uno no tiene estudios, cierto, tiene que ganar poco poh, porque si uno tiene estudios gana más... y de repente es eso, a lo mejor a mí me faltó eso, más estudios. Pero igual terminé mi cuarto medio. Eso es como un logro, porque la mayoría aquí está recién terminándolo aquí adentro, pero yo no poh. A lo mejor me faltó eso, seguir estudiando, hacer caso pa' tener una mejor vida".

Sin embargo, el proceso de socialización de un oficio o profesión no ocurre solo en los espacios educacionales formales, sino que también a través de las relaciones familiares y comunitarias. En el caso del micro-tráfico, este "oficio" se aprende precisamente en el marco de dichos vínculos. El relato de una reclusa de 30 años (ID8) da cuenta de dicho proceso de socialización:

"Afuera de la cárcel yo traficaba; mi marido, su familia, todos trafican, o sea, lo siguen haciendo... y yo estuve desde los 13 años con mi marido. Y ahora que cai presa recién nos 
separamos poh; él está en la calle, pero yo sola, pero en la calle yo traficaba. Y acá he trabajado en los talleres".

$\mathrm{Al}$ respecto, las parejas de mujeres micro-traficantes suelen ser agentes centrales en el proceso de aprendizaje de dicho oficio. Como indica una mujer privada de libertad de 19 años (ID15), la convivencia con su pareja constituye un momento central de su socialización en dicho oficio:

"Yo siempre he vivido con mi familia... fueron 3 años que vivi con mi pareja en la calle, que me fui con él, ahí me puse a traficar; tuve mis cosas igual, me fui con él poh y esa fue la única separación de mi familia que he tenido".

Como se ha mostrado, la trayectoria laboral individual está influenciada por la trayectoria laboral familiar (ilegal/criminal), habiendo una fusión entre individuo y familia en cuanto a este tipo de actividad. Sin embargo, este aprendizaje y sus consecuencias, no necesariamente han sido fáciles. Las historias laborales y familiares, en la mayoría de los casos entrevistados, están cruzadas de dolor y marginalización, como la de esta reclusa de 42 años (ID5):

"La feria, siempre la feria. La feria, con eso estaba igual, porque yo soy madre soltera de dos hijos, que ya fallecieron mis dos hijos, uno de 1 año y el otro de 15 años. Pero quedé con mi mamá y mi hermano que es inválido, que igual yo lo crié a él, porque en una separación que tuvieron mis padres yo quedé con los tres menores. Soy la mayor de todos mis hermanos. Somos 5. Y quedé criando a mis hermanos. Entonces igual tomé una responsabilidad muy fuerte a temprana edad, encargarme de mi hermano mientras mi papá se botó al alcohol y todo. Entonces yo quedé trabajando para ellos, trabajando para ellos hasta el día que murió mi último hijo de 15 años y me coloqué a traficar y llegué a este lugar, y de ahí no visto nunca más nada".

En conclusión, el sentido que adquiere el micro-tráfico para las mujeres dedicadas a esta actividad se encuentra estructurado en el marco del propio proceso de socialización a través del cual se aprende el oficio de micro-traficante. Así como el sistema educacional ha cumplido la función socializadora básica de la población con miras al mercado del trabajo y el trabajo 
asalariado, la comunidad y en especial, la familia y las parejas de estas mujeres han sido sus agentes socializadores para el aprendizaje de dicho oficio. Conocimientos y habilidades, así como orientaciones relativas a las acciones que buscan proveer a las personas de recursos materiales son aprendidos en este entorno educativo informal. Pese a que dicho proceso de formación puede ser comprendido desde el punto de vista societal como conducente hacia el desarrollo de comportamientos ilegales, desde el punto de vista subjetivo aquellos son “comportamientos desviados con intención integrativa" (Böhnisch, 1997: 33). En un contexto social neoliberal donde cada vez más se espera que las mujeres sean capaces de autosustentarse económicamente y de proveer recursos materiales para su hogar, situación especialmente evidente en los hogares monomarentales, dicho segmento de la población pareciera buscar su vía de integración a través de los oficios que le son posibles de ejercer.

\section{Del trabajo criminal al trabajo formal: ¿Para qué y quién trabajar?}

Así como ocurre en el sistema educacional, el proceso de formación y con ello de disciplinamiento constituye un objetivo central dentro de la cárcel (Foucault, 2002). En el caso del sistema penitenciario chileno, ambos procesos se llevan a cabo a través de cursos de nivelación escolar y talleres de capacitación. ${ }^{2}$ Otra instancia socializadora son los propios espacios de trabajo remunerado al interior del sistema penitenciario. ${ }^{3}$

En este contexto, el trabajo en el mundo penitenciario adquiere diversos sentidos para la población penitenciaria femenina. Desde el punto de vista de los estudios de género, la participación de las mujeres en la división del trabajo en la sociedad moderna supone una división sexual del trabajo, en donde las mujeres construyen su identidad a través de la

\footnotetext{
${ }^{2}$ Dichas funciones socializadoras son llevadas a cabo tanto por la administración penitenciaria como por diversas ONGs (por ej. Corporación Abriendo Puertas e INFOCAP) y grupos religiosos que ofrecen talleres de capacitación laboral y desarrollo personal (Abriendo Puertas, 2014; Cárdenas, 2011, 2013).

${ }^{3}$ Estos espacios son provistos generalmente por el sistema penitenciario mismo (por ej. lavanderías y cocinas de los centros penitenciarios), por las empresas externas o por las propias reclusas a través del autoempleo o el microemprendimiento y la contratación de reclusas por parte de otra reclusa (Cárdenas, 2010, 2011, 2013). En efecto, un número importante de participantes de este estudio reporta participación en actividades remuneradas dentro de la cárcel, tales como el Taller Minka (artesanía), Ventanas, Válvulas reguladores de gases, Guantes (peluquería) y Aseo Industrial (externo). Estas actividades regularían el tiempo de las reclusas, las orientarían hacia una tarea y les proveería de un ingreso mínimo.
} 
realización de tareas que involucran la entrega o el cuidado hacia otros (Beck y BeckGernsheim, 2003; Brunet Icart y Alarcón Alarcón, 2005). Así, para muchas reclusas el trabajo penitenciario es percibido como un medio para lograr el bienestar de otros, principalmente, de sus hijos/as. De esta manera, una de las razones principales para trabajar en la cárcel por parte de las reclusas sería la satisfacción de necesidades de los demás:

"Me gusta mucho trabajar, porque tengo un hijo de 7 años. Entonces pa' mandarle sus cositas y todo. Igual el niño necesita sus cosas, de ropa, de útiles de colegio, no sé poh, de aseo, eso". (ID11, 26 años)

"Algo muy importante poh, porque ayudo a mis hijos, me doy vueltas con esa plata, me compro mis cosas de aseo, ayudo a mi madre. Pa' mi el trabajo es algo importante, sobre todo en este lugar, porque estoy privada de libertad y hay muchas que no tienen visitas y yo a veces tampoco tengo visitas, tengo que pagar cosas, tengo que cuidar a mis hijos. Entonces feliz trabajar finalmente duro pa' poder ganar tu sueldo. De todo teni que hacer en la vida, nadie trabaja por ti. Si tu querí algo tení que trabajar por ello, si querí trabajar, trabaja, si querí comer que esforzarte por todo, todo. (...) es como un golpe bien fuerte a tu vida, porque yo antes era traficante igual, era atrevida, era peleadora cachai”. (ID29, 25 años)

Junto con la provisión de recursos materiales para otros, el trabajo penitenciario es percibido como un medio para estructurar la propia cotidianeidad. Específicamente, el trabajo penitenciario le permitiría a las reclusas regular sus horarios, lugares y vínculos sociales. De esta manera, el trabajo penitenciario podría promover un cambio del estado psicológico en que se encuentran, siendo una suerte de escape a la vida anterior. Por último, la estructura que provee el trabajo tendría un efecto preventivo frente al uso de drogas. Así lo manifiesta esta mujer privada de libertad de 27 años (ID12):

"Es que en los patios hay mucha droga. Yo estaba en la comunidad, en la comunidad yo me drogué y me bajaron y me iban a mandar a los patios y yo no me quería seguir volando. Entonces éste es un patio donde no hay drogas, donde puedo estar bien básicamente. Aquí es bueno, porque aquí tú, o sea yo, yo trabajo, llego a esta hora a almorzar, me voy a las 2, a las 5.30 llego, me baño, me visto y me voy a las 6 al colegio, 6.30. Entonces ahí llego a las 10 de la noche, me pongo el pijama y me acuesto y la luz ya está entera apagada a las 10 de la noche. En cambio en 
los patios no, en los patios te acostai a la hora que querí. (...) Trabajar para mí igual me ayuda, porque yo estaba con depresión, estaba muy mal y yendo a trabajar igual salí de esa depresión con puro trabajar y ponerle plata a mi hija, porque mi hija viene una vez al mes y así le tengo su plata y todo. Porque si ella viene no voy a tener plata, andaría en la volá o a lo mejor no vendria".

Adicionalmente, el trabajo penitenciario adquiere un sentido estructurador de la propia biografía. Específicamente, permitiría ordenar las acciones de las reclusas a lo largo de su vida. Así, trabajar es percibido por las reclusas como una actividad que les permite no solo ordenar su presente y sus acciones cotidianas, sino que también su tiempo futuro y las acciones que se pretende realizar en dicho momento. El trabajo penitenciario le permitiría por lo menos a algunas reclusas proyectar entonces su propia existencia más allá de la reclusión y dotar de sentido su tiempo presente, como lo explica esta mujer privada de libertad de 54 años (ID7):

"Trabajar significa que, yo me veo así, como que soy una persona que todavía puede salir adelante, que todavía puedo ser algo con mis hijos, que todavía valgo, que diga que tengo fuerzas para darme para sobrevivir. Y el trabajo aquí ayuda mucho, mucho, para salir uno de la depresión, porque te toma como una depresión encerrá, sale usted de una depresión, se ve bien. Pasa el tiempo más rápido, ya es distinto, yo avancé harto con el trabajo”.

De esta forma, el trabajo penitenciario otorga una atribución de sentido que se manifiesta en una mayor valoración personal y en la posibilidad de proyectarse laboralmente más allá de del periodo penitenciario. Así lo relata una reclusa de 28 años (ID1):

"Llegué acá condenada, imputada. Y me dijeron: sabi que acá dentro se puede trabajar; me dijeron: ¿querí trabajar? Y yo dije que sí. Sí tengo las ganas de trabajar, quiero ser otra persona en la vida, quiero salir adelante, voy a luchar por lo que quiero y lo voy a conseguir. Busqué mi trabajo y conseguí lo que quería. Aquí todavía sigo trabajando. Me levanto súper temprano a trabajar y hago las cosas que yo quiero. Me proyecto acá dentro y afuera también me proyecto, porque si tuve la capacidad de trabajar acá, puedo trabajar afuera”.

Por último, trabajar dentro del sistema penitenciario en Chile constituye un medio para acceder a los diversos beneficios penitenciarios, tales como los beneficios de salida. Es por esto 
que el trabajo en las cárceles suele ser entendido como un medio para hacer "conducta", es decir, para demostrarle al sistema penitenciario y a la sociedad que dicho individuo se comporta según las expectativas socialmente impuestas (Cárdenas, 2010, 2011, 2013). Así, en el caso de las mujeres privadas de libertad estudiadas, el trabajo suele constituirse en un medio para demostrar un comportamiento acorde a los principios del trabajo asalariado, como lo señala esta reclusa de 36 años (ID17):

“Conducta, más que nada conducta pa' ver la calle, pa' estar con tu familia poh, pa' que estén bien".

El hecho que el sentido del trabajo penitenciario para las reclusas no sea precisamente la obtención de dinero (como lo sería en parte en el trabajo formal y en el micro-tráfico), muestra una transformación de los medios y fines del trabajo. El objetivo ya no es el dinero, sino poder mantener los vínculos familiares. Por ejemplo, una mujer privada de libertad de 19 años (ID15) dice lo siguiente respecto a su trabajo penitenciario:

"Yo lo estoy haciendo por mi conducta, no para hacerme millonaria ni nada, yo lo hago por mi conducta porque mi familia me necesita. Por eso estudio en la noche y trabajamos de las 8.30 hasta las 12, a las 12 no sacan pa' acá a almorzar, después a las 2 nos vamos".

De esta manera, para las mujeres privadas de libertad el trabajo penitenciario se transforma en un medio de visibilización de acciones tendientes a "hacer conducta" y poder así recuperar su libertad y estar con su familia. Por ejemplo, así se expresa una mujer privada de libertad de 26 años (ID19) cuando se le pregunta por su actual trabajo en la cárcel:

"Mi familia es lo más importante que tengo, mi familia, estoy trabajando para, para irme luego pa' la calle, pero tengo que comportarme bien para salir de aqui (...) Para la conducta, si poh, para la conducta y para poder irse de aquí, pa' estar luego con la familia, andar trabajando pa' llevar plata pa' la casa. (...) De trabajar buena, muy buena, si porque sali de la rutina y te sirve harto, porque aparte que aqui podi salir pa' todos lados cuando trabajai, tení beneficios, teni buena conducta, te ayuda, ayuda mucho". 


\section{Comentarios finales: La diversidad en el orden social}

El trabajo asalariado se ha constituido en el referente normativo mediante el cual se han estructurado y estandarizado las acciones de los individuos tanto dentro como fuera del mercado del trabajo en el contexto de la configuración de la sociedad moderna. Sin embargo, la institución social del trabajo asalariado no sólo pareciera ser cuestionada por las transformaciones estructurales y organizacionales que los Estados y las empresas han venido emprendiendo en las últimas décadas para enfrentar los nuevos desafíos de un orden económico global, sino también por las propias acciones llevadas a cabo por los sujetos.

Al respecto, el micro-tráfico es una actividad económica debatible que atraviesa los países y por la cual muchas personas se encuentran recluidas en diversas cárceles a nivel mundial. En este artículo se ha puesto la atención en aquellas mujeres que han participado en actividades de micro-tráfico y que actualmente se encuentran recluidas en el Centro Penitenciario Femenino de Santiago de Chile, puesto que sus narraciones parecieran dar cuenta de la pluralidad de experiencias laborales que se despliegan más allá de los mandatos del trabajo asalariado.

Los resultados de este estudio indican que para estas reclusas el trabajo formal constituye un modo de integrarse al mercado del trabajo que, desde su punto de vista, supone gran sacrificio y escasa retribución económica. Dichos resultados son consistentes con las precarias condiciones en las cuales gran parte de las mujeres se ha venido integrando al mercado del trabajo en estas últimas décadas. Así, el micro-tráfico se presenta como un modo alternativo para mujeres de escasos recursos tanto para integrarse al mercado laboral como para continuar asumiendo las tareas de cuidado que tradicionalmente les han sido asignadas. Además, en torno al tráfico de estupefacientes es que se aprende un oficio, es decir, un conjunto de saberes y habilidades referidas a una actividad económica ilegal. La participación en dicha actividad les permite generar recursos económicos, pero al mismo tiempo las sitúa en una posición de subordinación como la ya vivida en relación a las tareas domésticas y familiares (relaciones de género) y/o sus experiencias laborales asalariadas previas (mercado laboral). 
La posición de subordinación laboral y familiar de estas mujeres difícilmente podrá ser modificada por el sistema carcelario. Esto, debido a que allí suelen ser socializadas bajo la lógica del trabajo asalariado sobre la base de una dinámica de subordinación múltiple: una subordinación productiva, en los mismos procesos productivos carcelarios, y una subordinación de libertad, a través del trabajo penitenciario a cambio de los diversos beneficios carcelarios. Pese a que las lógicas de la economía informal están siendo cada vez más normalizadas dentro de los espacios tradicionales de la economía formal, la discusión respecto a la creciente precarización sigue siendo un tema escasamente abordado en el debate público. En dicho contexto, así como las empresas y los Estados buscan encontrar la mejor de las posiciones posibles en esta nueva fase del capitalismo, cada individuo, con los recursos que tiene a la mano, busca también encontrar su lugar en la sociedad. Al igual que en los primeros momentos del proceso de industrialización, la cárcel vuelve a cumplir su función disciplinadora con todos/as aquellos/as quienes no acatan el orden social. En este sentido, en el contexto del capitalismo flexible las mujeres correspondientes a los sectores sociales más pobres son socializadas precisamente para una participación laboral funcional a los requerimientos de la fase actual del capitalismo, esto es, un uso precariamente flexible de la fuerza de trabajo. Al respecto, las actividades de micro-tráfico llevadas a cabo por parte de la población femenina pareciera ser un intento de cuestionamiento y desarrollo de estrategias de integración alternativas.

Por último, en este artículo hemos planteado cómo la situación de mujeres privadas de libertad por micro-tráfico cuestiona y al mismo tiempo reproduce el orden social, principalmente respecto a las relaciones de género y el significado del trabajo. En cuanto a las relaciones de género, las reclusas quebrarían el orden de género al no cumplir con las expectativas de su género respecto a los roles de cuidado; al mismo tiempo, se reproduciría el orden de género en el mercado de trabajo ilegal, ya que las mujeres suelen ocupar las posiciones inferiores dentro de la jerarquía de poder en el tráfico de estupefacientes. En cuanto al orden social, las reclusas vulnerarían la normativa social al cometer un delito. Asimismo, el hecho de ser mujeres pobres y de minorías étnicas quienes típicamente conforman la población penitenciaria femenina cuestionaría el orden social, pues mediante los mayores ingresos que perciben en relación a los trabajos remunerados a los que usualmente pueden acceder modifican la distribución de poder económico en esta sociedad. Considerando lo anterior, las 
mujeres privadas de libertad cuestionan el concepto tradicional de trabajo: las actividades económicas de micro-tráfico que realizaban "en la calle" son consideradas un trabajo, pues han sido un medio para cumplir con sus roles de cuidado y conseguir el sustento material para sus familias.

\section{Bibliografía}

- Anker, Richard (1997): "La segregación profesional entre hombres y mujeres. Repaso de las teorías". En: Revista Internacional del Trabajo, vol. 116, nº 3, pp. 343-370.

- Antony, Carmen (2003): "Panorama de la situación de las mujeres privadas de libertad en América Latina desde una perspectiva de género". En: DPLF et al. (eds.): Violencia contra las mujeres privadas de libertad en América Latina. México D.F.: Fundación para el Debido Proceso Legal (DPLF)/Comisión de Derechos Humanos del Distrito Federal (CDHDF)/Comisión Mexicana de Defensa y promoción de los Derechos Humanos (CMDPDH)/Open Society Institute, pp.75-90.

. (2007): "Mujeres invisibles: las cárceles femeninas en América Latina". En: Nueva Sociedad, n²08, pp. 73-85.

- Arriagada, Irma (2013): "Desigualdades en la familia: trabajo y cuidado en Chile". En Claudia Mora (ed): Desigualdad en Chile: La Continua Relevancia del Género. Santiago: Ediciones Universidad Alberto Hurtado, pp. 91-112.

- Arriagada, Irma y Hopenhayn, Martin (2000): "Producción, tráfico y consumo de drogas en América Latina”. En: Serie Políticas Sociales n40, División de Desarrollo Social, CEPAL, Santiago de Chile.

- Aulenbacher, Brigitte (2010): "Rationalisierung und der Wandel von Erwerbsarbeit aus der Genderperspektive”. En: Fritz Böhle, Gerd Günter Voß y Günther Wachtler (eds.), Handbuch Arbeitssoziologie: Wiesbaden: VS Verlag für Sozialwissenschaften, pp. 301-328.

- Bayón, María Cristina; Roberts, Bryan y Saraví, Gonzalo A. (1998): “Ciudadanía social y sector informal en América Latina”. En: Perfiles Latinoamericanos, 13, pp. 73-111.

- Beck, Ulrich y Beck-Gernsheim, Elisabeth (2003): La individualización. El individualismo institucionalizado y sus consecuencias sociales. Barcelona: Paidós. 
- Bolte, Karl Martin; Brater, Michael y Beck, Ulrich (1988): "Der Berufsbegriff als Instrument soziologischer Analyse". En: Karl Martin Bolte (ed.), Mensch, Arbeit und Betrieb, Weinheim: VCH-Verlag, pp. 39-54.

- Böhnisch, Lothar (1997): Sozialpädagogik der Lebensalter. Eine Einführung. Weinheim/München, Juventa.

- Brater, Michael (2010): “Beruflich Bildung”. En: Fritz Böhle, Gerd Günter Voß y Günther Wachtler (eds.), Handbuch Arbeitssoziologie: Wiesbaden: VS Verlag für Sozialwissenschaften, pp. 805-837.

- Brunet Icart, Ignasi y Alarcón Alarcón, Amado (2005): "Mercado de Trabajo y Familia". En: RIPS Revista de investigaciones políticas y sociológicas, vol. 4, nº 2, pp. 115-129.

- Cárdenas, Ana (2010): “Trabajo penitenciario en Chile”, GTZ-Ministerio de Justicia de Chile-ICSO, [en línea] Disponible en: http://www.icso.cl/wpcontent/uploads/2012/01/TRABAJO-PENITENCIARIO-EN-CHILE-versión-final-v2.pdf $[10 / 10 / 2011]$.

. (2011): "Mujeres y Cárcel en Chile”, GTZ-Ministerio de Justicia de Chile-ICSO, [en línea] Disponible en: http://www.icso.cl/wp-content/uploads/2012/01/Proyecto-GruposVulnerables-CPF-GIZ-MINJU-ICSO-versión-final-para-página-web-Diciembre-2011.pdf $[10 / 11 / 2012]$.

. (2013): "Género y precarización laboral: El trabajo penitenciario femenino en Chile". En: Claudia Mora (ed): Desigualdad en Chile: La Continua Relevancia del Género. Santiago: Ediciones Universidad Alberto Hurtado, pp. 143-169.

- Carpio, Jorge; Klein, Emilio y Novacovsky, Irene (eds.) (2000): Informalidad y Exclusión Social. Buenos Aires: Fondo de Cultura Económica, Siempro, OIT.

- Castel, Robert (1995): Les métamorphoses de la question sociale. Une chronique du salariat. Paris: Fayard.

- Cockburn, Cynthia (1983): Brothers: male dominance and technological change. London: Pluto Press.

- Comfort, Megan (2003): "In The Tube At San Quentin The "Secondary Prisonization" of Women Visiting Inmates”. En: Journal of Contemporary Ethnography vol. 32 no 1, pp. 77-107. - Corporación Abriendo Puertas (2014): Misión [en línea] www.abriendopuertas.cl [01/06/2014]. 
- Demszky von der Hagen, Alma y Voß, Gerd Günter (2010): “Beruf und Profession”. En: Fritz Böhle, Gerd Günter Voß y Günther Wachtler (eds.), Handbuch Arbeitssoziologie: Wiesbaden: VS Verlag für Sozialwissenschaften, pp. 751-803.

- DPLF et al. (2003): Violencia contra las mujeres privadas de libertad en América Latina. México D.F.: Fundación para el Debido Proceso Legal (DPLF)/Comisión de Derechos Humanos del Distrito Federal (CDHDF)/Comisión Mexicana de Defensa y promoción de los Derechos Humanos (CMDPDH)/Open Society Institute.

- Foucault, Michel (2002): Vigilar y castigar. Nacimiento de la Prisión. Buenos Aires: Siglo XXI Editores.

- Gendarmería de Chile (2008a): Compendio de Normativa Institucional Relativa a la Subdirección Técnica. Santiago de Chile: Gendarmería de Chile.

. (2008b): Compendio Estadístico. Subdirección Administrativa, Subdepartamento de Estadística y Control Penitenciario. Santiago de Chile: Gendarmería de Chile.

(2008c): Memoria 2008. Subdirección Administrativa, Subdepartamento de Estadística y Control Penitenciario. Santiago de Chile: Gendarmería de Chile.

- (2009): Estadísticas Oficiales de Gendarmería, [en línea] Disponible en: http://www.gendarmeria.cl/gendarmeriaweb/home.do [29/12/2008].

(2011): Memoria 2011. Subdirección Administrativa, Subdepartamento de Estadística y Control Penitenciario. Santiago de Chile: Gendarmería de Chile.

- Glucksmann, Miriam (1990): Women assemble: Women workers and the new industries in inter-war Britain. London \& New York: Routledge.

- Hoskyns, Catherine y Rai, Shirin M. (2007): "Recasting the Global Political Economy: Counting Women's Unpaid Work” En: New Political Economy vol. 12 n 3, pp. 297-317.

- Hughes, Christina (2002): Key concepts in feminist theory and research. London: Sage.

- INE (2014): “Metodología. Glosario. Departamento Estadísticas de Hogares”. Encuesta Nacional del Empleo, [en línea] Disponible en: http://www.ine.cl/canales/chile_estadistico/mercado_del trabajo/empleo/meto dologia/pdf/conceptos.pdf [8/02/2014].

- International Centre for Prison Studies (2012): World Female Imprisonment List, second edition. London: International Centre Prison Studies, King’s College London. 
- Jurczyk, Karin (2001): "Patriarchale Modernisierung. Entwicklungen geschlechtsspezifischer Arbeitsteilung im Zusammenhang mit der Entgrenzung von Öffentlichkeit und Privatheit”. En: Gabriele Sturm, Christina Schachtner, Renate Rausch y Karola Maltry (eds.): Zukunfts(t)räume. Geschlechterverhältnisseim Globalisierungsprozess, Königstein: Taunus, pp. 163-187.

- Kreisky, Eva (2000): “Geschlechtliche Fundierung von Politik und Staat”. En: Janshen, Doris (ed.), Blickwechsel. Der neue Dialog zwischen Frauen- und Männerforschung. Frankfurt/Main: Campus Verlag, pp. 167-192.

- LABORSTA (2014): LABORSTA Internet. Definitions, Main Statistics (annual), [en línea] Disponible en: http://laborsta.ilo.org/applv8/data/c2e.html [8/02/2014].

- OIT (1998): Chile: Crecimiento, empleo y el desafío de la justicia social. Santiago de Chile: Organización Internacional del Trabajo.

- Öztimur, Nese (2007): “Women as strategic agents of global capitalism”. En: International Review of Modern Sociology, vol. 33, n¹, pp. 117-128.

- Piras, Claudia (ed) (2006): Mujeres y trabajo en América Latina: Desafíos para las políticas laborales. Washington DC: Banco Interamericano de Desarrollo.

- PNUD (2010): Informe Desarrollo Humano en Chile. Género: Los desafíos de la Igualdad. Santiago de Chile: Programa de las Naciones Unidas para el Desarrollo.

- Portes, Alejandro; Castells, Manuel y Benton, Lauren A. (1989): The Informal Economy: Studies in Advanced and Less Developed Countries. Baltimore: The Johns Hopkins University Press.

- Portes, Alejandro (2000): "La economía informal y sus paradojas". En: Jorge Carpio, Emilio Klein e Irene Novacovsky, Informalidad y Exclusión Social. Buenos Aires/ México D.F.: SIEMPRO-Organización Internacional del Trabajo, Fondo de Cultura Económica, pp. 25-49.

- Portes, Alejandro y Hoffman, Kelly (2007): "Las estructuras de clase en América Latina: composición y cambios en la época neoliberal”. En: Rolando Franco, Arturo León y Raúl Atria (eds.), Estratificación y movilidad social en América Latina. Transformaciones estructurales de un cuarto de siglo. Santiago de Chile: LOM-CEPAL-GTZ, pp. 559-597. 
- Sassen, Saskia (2007a): "La conformación de los movimientos migratorios internacionales”. En: Sassen, Saskia (ed.) Una sociología de la globalización, Nueva York: Katz Editores, pp. 165-204.

. (2007b): "Nuevas clases globales". En: Sassen, Saskia, Una sociología de la globalización, Nueva York: Katz Editores, pp. 205-233.

- Sennett, Richard (1998): The corrosion of character. The personal consequences of work in the new capitalism. New York: W.W.Norton.

. (2005): The culture of the new capitalism. New Haven: Yale University Press.

- Torres Angarita, Andreina (2008): Drogas, cárcel y género en Ecuador: La experiencia de mujeres “mulas”. Tesis de maestría, FLACSO-Ecuador.

- Undurraga, Rosario (2011): Between family and work: women's participation in the labour market in Chile. Ph.D. tesis, University of Warwick, Coventry.

(2013): "Mujer y trabajo en Chile: ¿Qué dicen las mujeres sobre su participación en el mercado laboral?" En: Claudia Mora (ed.): Desigualdad en Chile: La Continua Relevancia del Género. Santiago: Ediciones Universidad Alberto Hurtado, pp. 113-141.

- Ungar, Mark (2003): "Prisons and Politics in Contemporary Latin America". En: Human Rights Quarterly, vol. 25, n 4, pp. 903-934.

- Valenzuela, Eduardo et al. (2012): "Impacto Social de la Prisión femenina en Chile”. En: Propuestas para Chile, Concurso de Políticas Públicas/2012, Santiago: Pontificia Universidad Católica de Chile.

- Voß, Gerd Günter (1997): Beruf und alltägliche Lebensführung - zwei subjektnahe Instanzen der Vermittlung von Individuum und Gesellschaft“. En: Gerd Günter Voß y Hans J. Pongratz (eds.), Subjektorientierte Soziologie, Opladen: Leske \& Budrich.

- Walby, Sylvia (1985): "Patriarchal Structures: the Case of Unemployment". En: Eva Gamarnikow, David Morgan, Jane Purvis y Daphne Taylorson (eds.) Gender, Class and Work, pp. 149-166.

. (1990): Theorizing patriarchy. Oxford: Basil Blackwell.

- Waring, Marilyn (2008): Counting for Something! Recognizing Women's Contribution to the Global Economy through Alternative Accounting Systems" En: Alison M. Jaggar (ed.) Just methods: an interdisciplinary feminist reader. Boulder: Paradigm, pp. 97-104. 